\title{
AN INEQUALITY BETWEEN THE PERMANENT AND THE DETERMINANT
}

\author{
P. M. GIBSON
}

A number of relationships between the permanent and other matrix invariants have been discovered [3]. In this paper we prove an inequality between the permanent and the determinant of $I-A$, where $I$ is the $n$-square identity matrix and $A$ is an $n$-square substochastic matrix.

Suppose that $A=\left[a_{i j}\right]$ is an $n$-square matrix. The permanent of $A$ is defined by

$$
\text { per } A=\sum a_{1 i_{1}} a_{2 i_{2}} \cdots a_{n i_{n}},
$$

where the summation is over all permutations $i_{1} \cdots i_{n}$ of $1 \cdots n$. If $a_{i j} \geqq 0$ and each row sum of $A$ is no greater than 1 , then $A$ is a substochastic matrix. If $A$ is substochastic with each row sum equal to 1 , then $A$ is a stochastic matrix.

If $r$ is an integer, $1 \leqq r<n$, let $Q_{r, n}$ denote the set of all sequences $\omega=\left(\omega_{1}, \omega_{2}, \cdots, \omega_{r}\right)$ of integers for which $1 \leqq \omega_{1}<\omega_{2}<\cdots<\omega_{r} \leqq n$. If $A$ is an $n$-square matrix and $\omega \in Q_{r, n}$ then $A_{\omega}$ is the $(n-r)$-square submatrix of $A$ that remains after rows and columns $\omega_{1}, \cdots, \omega_{r}$ are removed.

The following theorem has been proved by the author in these Proceedings [2] and by Brualdi and Newman [1].

Theorem 1. If $A$ is a substochastic matrix, then per $(I-A) \geqq 0$.

We use Theorem 1 and mathematical induction to prove the following.

THEOREM 2. If $A$ is an $n$-square substochastic matrix, then $\operatorname{per}(I-A)$ $\geqq \operatorname{det}(I-A) \geqq 0$.

Proof. Clearly, the theorem is true for $n=1$. Let $A$ be an $m$-square substochastic matrix and assume that Theorem 2 is true for all $n$, $1 \leqq n<m$. Let $r_{i}$ be equal to the $i$ th row sum of $A, i=1, \cdots, m$. Define the $m$-square matrix $D=\left[d_{i j}\right]$ by

$$
\begin{aligned}
d_{i j} & =d_{j}=1-r_{j} \text { if } i=j, \\
& =0 \text { if } i \neq j .
\end{aligned}
$$

Received by the editors April 10, 1967. 
Obviously $D$ is a nonnegative diagonal matrix and $B$ is a stochastic matrix, where $B=D+A$. We have

$$
I-A=D+(I-B) \text {. }
$$

It is well known that

$$
\begin{aligned}
\operatorname{det}[D+(I-B)]= & d_{1} d_{2} \cdots d_{m}+\operatorname{det}(I-B) \\
& +\sum_{r=1}^{m-1} \sum_{\omega \in Q_{r, m}} d_{\omega_{1}} d_{\omega_{2}} \cdots d_{\omega_{r}} \operatorname{det}(I-B)_{\omega} .
\end{aligned}
$$

It is easy to prove a similar expansion for the permanent,

$$
\begin{aligned}
\operatorname{per}[D+(I-B)]= & d_{1} d_{2} \cdots d_{m}+\operatorname{per}(I-B) \\
& +\sum_{r=1}^{m-1} \sum_{\omega \in Q_{r, m}} d_{\omega_{1}} d_{\omega_{2}} \cdots d_{\omega_{r}} \operatorname{per}(I-B)_{\omega} .
\end{aligned}
$$

Since each row sum of $I-B$ is zero, the columns of $I-B$ are linearly dependent and

$$
\operatorname{det}(I-B)=0 .
$$

According to Theorem 1,

$$
\operatorname{per}(I-B) \geqq 0 \text {. }
$$

Since each square submatrix of a stochastic matrix is substochastic, by the inductive assumption,

$$
\operatorname{per}(I-B)_{\omega} \geqq \operatorname{det}(I-B)_{\omega} \geqq 0
$$

for every $\omega \in Q_{r, m}, r=1, \cdots, m-1$. Hence

$$
\begin{gathered}
\operatorname{per}(I-A) \geqq \operatorname{det}(I-A) \geqq 0 . \\
\text { REFERENCES }
\end{gathered}
$$

1. R. A. Brualdi and M. Newman, Proof of a permanental inequality, Quart. J. Math. Oxford Ser. (2) 17 (1966), 234-238.

2. P. M. Gibson, A short proof of an inequality for the permanent function, Proc. Amer. Math. Soc. 17 (1966), 535-536.

3. M. Marcus and H. Minc, Permanents, Amer. Math. Monthly 72 (1965), 577591.

North Carolina State University, Raleigh and

University of Alabama, Huntsville 